\title{
The Optical Transfer Function of a Diffraction-limited System for Polychromatic Illumination
}

\author{
Muralidhara Subbarao \\ State University of New York at Stony Brook \\ Department of Electrical Engineering \\ Stony Brook, NY 11794-2350
}

\begin{abstract}
A closed-form expression is derived for the optical transfer function of a diffraction limited system under polychromatic illumination. The expression is useful in a method for the accurate and efficient computation of the optical transfer function of an optical system. The expression is also useful in the theoretical analyses of the optical system. This method is illustrated with an example where the optical transfer function of the human eye is computed.
\end{abstract}




\section{Introduction}

During our recent work in the area of machine vision on a new method for finding the distance of objects [1], [2], it became necessary for us to know the optical transfer function (OTF) of a camera under ambient illumination such as white light. A search by us to find an analytic model of the white-light OTF in the optics literature was unsuccessful, although substantial literature was found on OTF for monochromatic light. A twodimensional Gaussian has sometimes been suggested as a possible model for the OTF of a camera [3], [4], but only as an approximation; a theoretical model appears not to have been derived. This motivated the current work.

An analytic expression is known in optics for the OTF of a diffractionlimited system for incoherent monochromatic illumination. Here this expression is integrated with respect to wave length to obtain an explicit expression for the OTF under polychromatic illumination. The integration of the original expression was performed with the help of MACSYMA [5], a computer program that can perform complex symbolic manipulations on mathematical expressions. (MACSYMA is available on most main-frame computers and is easy to use. See Appendix A.)

The OTF derived here is useful in the efficient estimation of the OTF of an optical system. This method is illustrated with an example. First we con- 
sider the OTF of a system with circular exit pupil. Section III deals with the case of a square exit pupil.

\section{Derivation of OTF: circular exit pupil}

The geometry of an aberration-free diffraction limited system with a circular exit pupil is shown in Fig. 1 . In this figure, $l$ is the diameter of the aperture and $d_{i}$ is the distance between the aperture and the image detector plane. The OTF of such a system for monochromatic incoherent light of wave length $\lambda$ is given in Goodman [6] to be

$H(\rho, \lambda)= \begin{cases}\frac{2}{\pi}\left[\cos ^{-1}\left(\frac{\rho}{2 \rho_{0}}\right)-\frac{\rho}{2 \rho_{0}} \sqrt{1-\left(\frac{\rho}{2 \rho_{0}}\right)^{2}}\right] & \begin{array}{l}\text { for } \rho \leq 2 \rho_{0} \\ 0\end{array} \\ \text { otherwise }\end{cases}$

where $\rho$ is the spatial frequency in polar coordinates ( $H$ is circularly symmetric, and hence independent of $\theta$ ). The quantity $\rho_{0}$ is given by

$$
\rho_{0}=\frac{l}{2 \lambda d_{i}} .
$$

Note that $2 \rho_{0}$ is the spatial cutoff frequency of the system. (Interestingly, the spatial cutoff frequency of the same system under coherent illumination is only half of this, i.e. $\rho_{0}[6]$. Therefore incoherent illumination may often be more desirable than coherent illumination.) Fig. 2 shows $H(\rho, \lambda)$ for a particular $\lambda$. In order to find the OTF for white light with a constant spectral 
distribution, equation (1) should be integrated with respect to the wave length $\lambda$. Simple integration without regard to the phase of electromagnetic field distribution is justified because, for incoherent illumination, the imaging system can be treated as a linear mapping of light intensity [6].

Using the notation

$$
t=\frac{\rho d_{i}}{l},
$$

$H(\rho, \lambda)$ in equation (1) can be expressed as

$H(\rho, \lambda)= \begin{cases}\frac{2}{\pi}\left[\cos ^{-1}(t \lambda)-(t \lambda) \sqrt{1-(t \lambda)^{2}}\right] & \text { for } t \leq \frac{1}{\lambda} \\ 0 & \text { otherwise. }\end{cases}$

The MACSYMA symbolic integration program was used to integrate the above expression for $H$ with respect to $\lambda$. The indefinite integral is

$$
\begin{aligned}
& G_{0}(\rho, \lambda)=\int H(\rho, \lambda) d \lambda \\
&=\frac{2}{\pi} {\left[\int \cos ^{-1}(t \lambda) d \lambda-t \int \lambda \sqrt{1-(t \lambda)^{2}} d \lambda\right] } \\
&=\frac{2}{\pi}\left[\lambda \cos ^{-1}(t \lambda)-\frac{\sqrt{1-(t \lambda)^{2}}}{t}+\frac{\left(1-(t \lambda)^{2}\right]^{\frac{3}{2}}}{3 t}\right] .
\end{aligned}
$$

The derivative of the above expression can be easily verified to be the original expression in equation (4). Now we consider the actual estimation of the OTF using the above expression. 
For an optical system (e.g. Fig. 1) let $S(\lambda)$ be the normalized spectral distribution of the object radiance, $M(\lambda)$ be the effective normalized spectral transmittance of all the media through which the light originating from the object and incident on the image detector traverses, and $R(\lambda)$ be the normalized spectral response of the image detector. Then, the overall normalized weighting function $P(\lambda)$ is defined by

$$
P(\lambda)=S(\lambda) M(\lambda) R(\lambda),
$$

and the effective OTF of the system is given by

$$
O(\rho)=\frac{1}{A} \int_{-\infty}^{\infty} P(\lambda) H(\rho, \lambda) d \lambda,
$$

where

$$
A=\int_{-\infty}^{\infty} P(\lambda) d \lambda
$$

We next consider the derivation of $O(\rho)$ for two different models of $P(\lambda)$ and then mention how the case of a general $P(\lambda)$ can be handled by a piecewise polynomial approximation.

Example 1. Constant model of $P(\lambda)$ :

Consider the case where $P(\lambda)$ is uniform in an interval $\left[\lambda_{\min }, \lambda_{\max }\right]$. Let

$$
P(\lambda)=\left\{\begin{array}{l}
1 \text { for } \lambda_{\min } \leq \lambda<\lambda_{\max } \\
0 \text { otherwise }
\end{array} .\right.
$$

Referring to Fig. 3, we define the following quantities: 


$$
\begin{gathered}
\rho_{\min }=\frac{l}{\lambda_{\max } d_{i}}, \rho_{\max }=\frac{l}{\lambda_{\min } d_{i}}, \\
\rho_{\min } \leq \rho,<\rho_{\max } \text { and } \lambda,=\frac{l}{\rho^{\prime} d_{i}} .
\end{gathered}
$$

Note that, $\rho_{\min }, \rho_{\max }$, and $\rho$, are the spatial cutoff frequencies of the system for wave lengths $\lambda_{\max }, \lambda_{\min }$, and $\rho$ 'respectively. In particular, it should be noted that, the cutoff frequencies decrease with increasing wave lengths.

Now the OTF is obtained by evaluating the definite integral

$$
O(\rho)= \begin{cases}\frac{1}{\lambda_{\max }-\lambda_{\min }}\left[G_{0}(\rho, \lambda)\right]_{\lambda_{\min }}^{\lambda_{\max }} & \\ \frac{1}{\lambda_{\max }-\lambda_{\min }}\left[G_{0}(\rho, \lambda)\right]_{\lambda_{\min }}^{\lambda=l /\left(\rho d_{i}\right)} & \text { for } 0 \leq \rho<\rho_{\min } \\ 0 & \text { for } \rho_{\min } \leq \rho=\rho,<\rho_{\max }\end{cases}
$$

Fig. 3 is helpful in understanding why the limits of evaluation of the definite integral are different in the above expression. A brief explanation is given in the next paragraph.

In Fig. 3, if $\rho=\rho_{1}$, where $0 \leq \rho_{1}<\rho_{\min }$, it is clear that $H(\rho, \lambda)$ is nonzero for all wave lengths in the interval $\left[\lambda_{\min }, \lambda_{\max }\right]$. Therefore the limits of integration are $\lambda_{\min }$ and $\lambda_{\max }$. If $\rho=\rho^{\prime}$ where $\rho_{\min } \leq \rho^{\prime}<\rho_{\max }$, then $H(\rho, \lambda)$ is non-zero only for those wave lengths lying in the interval $\left[\lambda_{\min }, \lambda_{\prime}\right]$. Hence the limits $-\lambda_{\min }$ and $\lambda$, If $\rho=\rho_{2}$, where $\rho_{2}>\rho_{\max }$, then $H(\rho, \lambda)$ is zero for all $\lambda$ in the interval $\left[\lambda_{\min }, \lambda_{\max }\right]$. 
The same limits used in the evaluation of the definite integral in equation (10) will appear twice more in this paper. A clear understanding of them now will be of much help later. Therefore we urge the reader to pause for a moment and be convinced of their correctness.

Note that, except at the two points $\rho_{\min }$ and $\rho_{\max }$, the function $O(\rho)$ above is continuous and differentiable everywhere.

In the above example we have assumed $P(\lambda)$ to be a constant in the interval $\left[\lambda_{\min }, \lambda_{\max }\right]$. If $P(\lambda)$ is not constant, then the above method can still be used by approximating $P(\lambda)$ by a function that is piecewise constant in small intervals. Indeed we can do better than this; we found (using MACSYMA) that not only $H(\rho, \lambda)$, but also $\lambda^{n} H(\rho, \lambda)$ is also integrable in closed-form for at least $n=0,1,2,3,4,5,6$. (We suspect that it is integrable for all positive integer values of $n$, but we did not investigate this further because in most practical applications the values 0 to 6 may suffice.) Therefore, an arbitrary function $P(\lambda)$ can be approximated by a piecewise polynomial of order up to six and the OTF can be computed very efficiently. In particular, piecewise linear approximation may suffice in most practical applications. The relevant integral, obtained using MACSYMA, is given below.

$G_{1}(\rho, \lambda)=\int \lambda H(\rho, \lambda) d \lambda$ 


$$
\begin{aligned}
& =\frac{2}{\pi}\left[\int \lambda \cos ^{-1}(t \lambda) d \lambda-t \int \lambda^{2} \sqrt{1-(t \lambda)^{2}} d \lambda\right] \\
& =\frac{2}{\pi}\left[\frac{\sin ^{-1}(t \lambda)}{8 t^{2}}-\frac{3 \lambda \sqrt{1-(t \lambda)^{2}}}{8 t}+\frac{\lambda^{2} \cos ^{-1}(t \lambda)}{2}+\frac{\lambda\left(1-(t \lambda)^{2}\right)^{\frac{3}{2}}}{4 t}\right] .
\end{aligned}
$$

We illustrate the use of the above equation to obtain the OTF with an example next.

Example 2. Linear model of $P(\lambda)$ :

We used equation (11) above to compute the OTF of the human eye. The $P(\lambda)$ corresponding to the human eye is shown in Fig. 4. The interval in which $P(\lambda)$ is non-zero was divided into seven intervals at $\lambda_{i}$ for $i=1,2, \ldots, 8$. In each interval the function was taken to be linear of the form: $P(\lambda)=m_{i} \lambda+c_{i}$ for $i=1,2, . ., 7$. The approximate values of $\lambda_{i}, m_{i}$, and $c_{i}$ are given in Table 1.

\section{Let}

$G(\rho, \lambda)=\int P(\lambda) H(\rho, \lambda) d \lambda$.

For a piecewise linear approximation of $P(\lambda)$ we have

$$
\begin{array}{r}
G(\rho, \lambda)=\int\left(m_{i} \lambda+c_{i}\right) H(\rho, \lambda) d \lambda \\
=m_{i} G_{1}(\rho, \lambda)+c_{i} G_{0}(\rho, \lambda),
\end{array}
$$

where $G_{0}, G_{1}$ are as in equations (5) and (11) respectively. In each interval $\left[\lambda_{i}, \lambda_{i+1}\right]$, the definite integral is evaluated using rules similar to those in 
equation (10). First we let $\lambda_{i}=\lambda_{\min }, \lambda_{i+1}=\lambda_{\max }$ and define $\rho_{\min }, \rho_{\max }, \rho^{\prime}$, and $\lambda$, as in equations $(9 a-d)$. Then

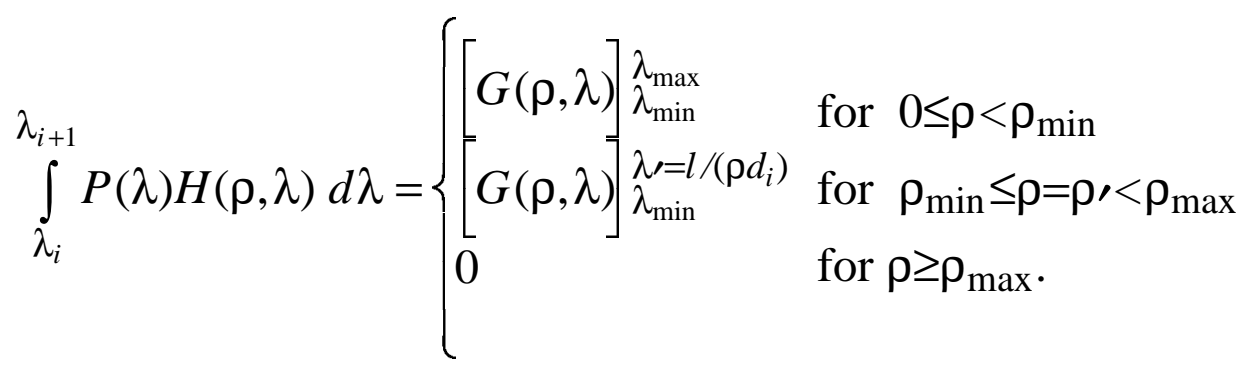

From this expression, the OTF is computed as

$$
O(\rho)=\frac{1}{A} \sum_{i=1}^{m} \int_{\lambda_{i}}^{\lambda_{i+1}} P(\lambda) H(\rho, \lambda) d \lambda
$$

where

$$
A=\sum_{i=1}^{m} \int_{\lambda_{i}}^{\lambda_{i+1}} P(\lambda) d \lambda
$$

and $m=7$. The resulting normalized OTF for the human eye is shown in Fig. 5. This method of computing OTF should be compared to the experimental methods referred to in [7].

Equations (12), (13) and (14) above are actually valid for arbitrary $P(\lambda)$; they are not restricted to a piecewise linear model of $P(\lambda)$. Therefore they can be used even when $P(\lambda)$ is approximated by a piecewise polynomial function (at least up to order six). Some useful integrals (obtained using MACSYMA) for this purpose are given in Appendix B. The OTF $O(\rho)$ defined by an equation such as equation (14a) is analytic everywhere 
except at a small finite number of points corresponding to $\lambda_{i}$.

The expressions for OTFs in equations (10) and (14) are valid only for the case of an aberration-free diffraction limited system. If aberrations are present, then the expressions will be different.

Till now we have described a method for finding the OTF of an optical system with a circular aperture. Analogous results can be derived for the case of square aperture. The integral in this case is much simpler than that for a circular aperture. We consider this next.

\section{Derivation of OTF: square exit pupil}

Let $l$ be the width of the square aperture of an optical system and, as before, let $d_{i}$ be the distance between the aperture and the image detector. For a diffraction-limited system, the OTF for incoherent monochromatic light illumination is given in [6] to be

$H\left(f_{x}, f_{y}, \lambda\right)= \begin{cases}\left(1-\frac{\lambda d_{i}\left|f_{x}\right|}{l}\right)\left[1-\frac{\lambda d_{i}\left|f_{y}\right|}{l}\right) & \text { for }\left|f_{x}\right| \leq \frac{l}{\lambda d_{i}},\left|f_{y}\right| \leq \frac{l}{\lambda d_{i}} \\ 0 & \text { otherwise }\end{cases}$

In the above expression, $f_{x}, f_{y}$ are the spatial frequencies along the $x$ and $y$ axes respectively. This OTF has the form of a pyramid as shown if Fig. 6. This OTF is symmetric in the four quadrants of the $f_{x}, f_{y}$ plane. Therefore the OTF for white light also will be symmetric in the four quadrants. 
Because of this symmetry, we will consider finding the OTF in only the first quadrant corresponding to $f_{x} \geq 0$ and $f_{y} \geq 0$. Having determined the OTF in one quadrant, the OTF in the other quadrants is determined through mirror reflection. Therefore we have

$H\left(f_{x}, f_{y}, \lambda\right)= \begin{cases}{\left[1-\frac{\lambda d_{i} f_{x}}{l}\right]\left[1-\frac{\lambda d_{i} f_{y}}{l}\right]} & \text { for } 0 \leq f_{x} \leq \frac{l}{\lambda d_{i}}, 0 \leq f_{y} \leq \frac{l}{\lambda d_{i}} \\ 0 & \text { otherwise. }\end{cases}$

It is trivial to integrate the above expression with respect to $\lambda$. Further, $\lambda^{n} H\left(f_{x}, f_{y}, \lambda\right)$ can also be integrated easily for $n=0,1,2,3, \ldots$. Therefore, $P(\lambda)$ can be approximated by a piecewise polynomial of any order in a given interval. Let

$$
G\left(f_{x}, f_{y}, \lambda\right)=\int P(\lambda) H\left(f_{x}, f_{y}, \lambda\right) d \lambda .
$$

Now, for any interval $\left[\lambda_{i}, \lambda_{i+1}\right]$, define $\lambda_{\min }=\lambda_{i}, \lambda_{\max }=\lambda_{i+1}$ and the quantities $\rho_{\min }, \rho_{\max }, \rho^{\prime}, \lambda$, exactly as in equations (9a-d). Also, define a new quantity $\rho$ to be the maximum of $f_{x}$ and $f_{y}$ (reminder: $f_{x} \geq 0$ and $f_{y} \geq 0$ assumed due to symmetry), i.e.

$$
\rho=\operatorname{maximum}\left(f_{x}, f_{y}\right) .
$$

Now the required definite integral is given by an equation that is almost exactly the same as equation (13); only the arguments of $G$ and $H$ need to be changed from $(\rho, \lambda)$ to $\left(f_{x}, f_{y}, \lambda\right)$. We repeat it here for clarity: 


$$
\int_{\lambda_{i}}^{\lambda_{i+1}} P(\lambda) H\left(f_{x}, f_{y}, \lambda\right) d \lambda= \begin{cases}{\left[G\left(f_{x}, f_{y}, \lambda\right)\right]_{\lambda_{\min }}^{\lambda_{\max }}} & \text { for } 0 \leq \rho<\rho_{\min } \\ {\left[G\left(f_{x}, f_{y}, \lambda\right)\right]_{\lambda_{\min }}^{\lambda=l /\left(\rho d_{i}\right)}} & \text { for } \rho_{\min } \leq \rho=\rho,<\rho_{\max } \\ 0 & \text { for } \rho \geq \rho_{\max } . \quad \text { (19) }\end{cases}
$$

The actual OTF can then be obtained using the following equation which is similar to equation $(14 \mathrm{a})$ :

$$
O\left(f_{x}, f_{y}\right)=\frac{1}{A} \sum_{i=1}^{m} \int_{\lambda_{i}}^{\lambda_{i+1}} P(\lambda) H\left(f_{x}, f_{y}, \lambda\right) d \lambda
$$

where $A$ is given by equation (14b) and $m$ is the number of intervals.

\section{Conclusion}

An analytic expression is derived for the OTF of a diffraction-limited system for polychromatic illumination. The expression has been derived for circular and square exit pupils. It may also be possible to derive such analytic expressions for many other shapes of exit pupils. The steps would be similar to the case of circular (or square) exit pupil. This work is useful in modeling the OTF of an optical instrument.

Acknowledgements: I thank Prof. Joseph W. Goodman at Stanford and Prof. Harbans Dhadwal at Stony Brook for helpful discussions. Mr. Rajiv Mahajan helped with an early version of a computer program to compute and plot the OTF. This research was supported in part by the National Sci- 
ence Foundation (IRI-8821923), and by the Olympus Corporation.

\section{Appendix A. MACSYMA}

The information in this appendix on MACSYMA has been included as per the suggestion of one of the referees of this paper. This information is taken from the online manual of our computer system (VAX 11/780, Department of Electrical Engineering, State University of New York at Stony Brook; October 1989).

\section{Description:}

MACSYMA is a large computer programming system written in LISP. It is used for performing symbolic as well as numerical mathematical manipulations. With MACSYMA the user can differentiate, integrate, take limits, solve systems of linear or nonlinear equations, expand functions in Laurent or Taylor series, solve differential equations (using direct or transform methods), compute Poisson series, plot curves, and manipulate matrices and tensors. There is a MACSYMA newsletter which is published every two months. You can see your computer site manager for information on this.

\section{History:}

MACSYMA is a large symbolic manipulation program developed by the 
Mathlab Group of the MIT Laboratory for Computer Science. Since 1982 MACSYMA has been supported and distributed by Symbolics, Inc. of Cambridge, Mass. MACSYMA is a trademark of Symbolics, Inc.

\section{Appendix B. Some Useful Integrals}

Let $H(\rho, \lambda)$ be as defined by equations (4) and (3). Then the following integrals will be useful in obtaining OTF by modeling $P(\lambda)$ by piecewise quadratic and cubic polynomials. These integrals were obtained using MACSYMA.

$$
\begin{gathered}
\int \lambda^{2} H(\rho, \lambda) d \lambda=\frac{\lambda^{3} \cos ^{-1}(\lambda t)}{3}+\frac{\lambda^{2}\left(1-(\lambda t)^{2}\right)^{\frac{3}{2}}}{5 t}+\frac{2\left(1-(\lambda t)^{2}\right)^{\frac{3}{2}}}{15 t^{3}} \\
-\frac{\lambda^{2} \sqrt{1-(\lambda t)^{2}}}{9 t}-\frac{2 \sqrt{1-(\lambda t)^{2}}}{9 t^{3}} . \\
\int \lambda^{3} H(\rho, \lambda) d \lambda=-\frac{\sin ^{-1}(\lambda t)}{32 t^{4}}-\frac{\lambda^{3} \sqrt{1-(\lambda t)^{2}}}{16 t}-\frac{5 \lambda \sqrt{1-(\lambda t)^{2}}}{32 t^{3}} \\
+\frac{\lambda^{3}\left(1-(\lambda t)^{2}\right)^{\frac{3}{2}}}{6 t}+\frac{\lambda\left(1-(\lambda t)^{2}\right)^{\frac{3}{2}}}{8 t^{3}}+\frac{\lambda^{4} \cos ^{-1}(\lambda t)}{4} .
\end{gathered}
$$

As mentioned in the main text, $\lambda^{n} H(\rho, \lambda)$ is integrable for at least $n=0,1,2, . ., 6$. Therefore if one wishes to use a polynomial model of order more than three for $P(\lambda)$ then we suggest the use of MACSYMA to get the integrals. We do not list the integrals here for $n=4,5,6, \ldots$ as the expressions 
are cumbersome and their use in practical applications is questionable.

\section{References}

1. M. Subbarao, "Method and apparatus for determining the distances between surface patches of three-dimensional spatial scene and a camera system," U.S. patent application no. 126407 (pending), November (1987).

2. M. Subbarao, "Parallel depth recovery by changing camera parameters,' Proceedings of the Second International Conference on Computer Vision, Florida, USA, pp. 149-155, December (1988).

3. B. K. P. Horn, Robot Vision, McGraw-Hill Book Company, pp. 126, (1986).

4. W. F. Schreiber, Fundamentals of Electronic Imaging Systems, Springer-Verlag, Section 2.5.2., (1986).

5. W. A. Martin and R. J. Fateman, "The MACSYMA system,' Proc. ACM 2d Symposium on Symbolic and Algebraic Manipulation, Los Angeles, CA, pp. 23-25, (1971). 
6. J. W. Goodman, Introduction to Fourier Optics, McGraw-Hill Book Company, pp. 118-120, (1968).

7. R. W. Gubisch, “Optical performance of the human eye,' Journal of the Optical Society of America, Vol. 57, No. 3, pp. 407-415, March (1967). 
Fig. 1. A diffraction-limited system with circular exit pupil.

Fig. 2. OTF of a diffraction-limited system with a circular exit pupil under incoherent quasimonochromatic illumination (after Goodman [6]).

Fig. 3. Determining the limits for the evaluation of the definite integrals.

Fig. 4. Approximate visibility curve for a normal eye under white light illumination (see Schreiber [4]).

Fig. 5. OTF of a diffraction limited normal eye under white light illumination.

Fig. 6. OTF of a diffraction-limited system with a square exit pupil under incoherent monochromatic illumination (after Goodman [6]). 
center $\operatorname{delim()}$ box $; 1|\mathrm{c}| \mathrm{c}|\mathrm{c}| \mathrm{c}|\mathrm{c}| \mathrm{c}|\mathrm{c}| \mathrm{c} 1|\mathrm{c}| \mathrm{c}|\mathrm{c}| \mathrm{c}|\mathrm{c}| \mathrm{c}|\mathrm{c}| \mathrm{c} 1|\mathrm{c}| \mathrm{c}|\mathrm{c}| \mathrm{c}|\mathrm{c}|$ $\begin{array}{ccccccccccccccccccccccccc}\mathrm{c} & \mid & \mathrm{c} & \mid & \mathrm{c} & 1 & \mid & \mathrm{c} & \mid & \mathrm{c} & \mid & \mathrm{c} & \mid & \mathrm{c} & \mid & \mathrm{c} & \mid & \mathrm{c} & \mid & \mathrm{c} & \mid & \mathrm{c} & 1 & 1 & 1 \\ i: & & 1 & 2 & & 3 & & 4 & & 5 & & 6 & & 7 & & 8 & & & & \lambda_{i} & & \text { in }\end{array}$ $A^{0}: \quad \begin{array}{llllllllllllll} & 4250 & 4750 & 5000 & 5350 & 5550 & 5700 & 6500 & 6850^{-} & \lambda_{i} & \text { in }\end{array}$ $\begin{array}{llllllllllll}m_{i}: & 0.020 & 0.088 & 0.180 & 0.025 & -0.020 & -0.109 & -0.029 & - & c_{i}: & -85.0 & -\end{array}$

Table 1. 